\title{
Practice Spotlight: Interdisciplinary HIV Outpatient Clinic
}

\author{
Christine Hughes, BScPharm, PharmD, \\ ACPR, FCSHP \\ Associate Professor \\ Faculty of Pharmacy and Pharmaceutical \\ Sciences \\ University of Alberta \\ Clinical Pharmacotherapy Practitioner, HIV \\ Northern Alberta HIV Program and \\ Alberta Health Services \\ Edmonton, Alberta
}

$\mathrm{T}$ he Northern Alberta HIV Program is delivered by an interdisciplinary team that cares for HIV-infected adults and children living in the northern half of the province. The program serves a diverse population, many of whom represent marginalized groups, for whom there are significant challenges in providing patient care. In particular, addictions, mental illness, language barriers, cultural barriers, and homelessness are common within this patient population. The team consists of pharmacists, nurses, social workers, adult and pediatric infectious disease specialists, psychologists, and a dietician.

This HIV program was established nearly 20 years ago and was based at the time at the University of Alberta Hospital in Edmonton. The original team consisted of infectious disease specialists, a social worker, and a nurse practitioner. As the complexity of antiretroviral drug therapy increased, Dr Stan Houston, director of the Northern Alberta HIV Program, advocated to Capital Health's Regional Pharmacy Services (now part of Alberta Health Services) for a pharmacist to join the team. This effort was successful, and in 1998 Christine Hughes became the program's first pharmacist. The pharmacist position was established as a cross-appointment between the University of Alberta's Faculty of Pharmacy and Pharmaceutical Sciences and Regional Pharmacy Services.

HIV-infected patients are typically referred to the Northern Alberta HIV Program by their primary care providers. A program nurse then triages the cases by complexity and acuity. At their first clinic visit, patients undergo a comprehensive assessment by team members, which includes social and medical history, medication and allergy history, physical examination, review of laboratory results, and education about HIV disease and its transmission. For patients who are candidates for initiation of antiretroviral therapy, a physician and a pharmacist discuss treatment options in relation to patient-specific factors, such as comorbid illnesses, concomitant medications, and baseline resistance, and work with the patient to establish a plan to initiate therapy. The pharmacist then meets with the patient to discuss previous medication experiences, the goals of therapy, and the possible side effects and their management, and to answer the patient's questions. Given the complex needs of this patient population, the pharmacist must work closely with a nurse and a social worker to optimize successful implementation of the drug therapy. For example, the team considers and discusses each patient's potential need for adherence aids, directly observed therapy, medication coverage for non-HIV-related medications, and involvement of community programs or community pharmacies to provide support.

In terms of ongoing patient care, the pharmacist monitors laboratory results and other signs and symptoms to identify any adverse effects of antiretroviral drug therapy that may arise. The pharmacist will also initiate therapy to manage side effects such as nausea and diarrhea, if appropriate. Because antiretroviral therapy often has metabolic side effects, including hyperlipidemia, the pharmacist also performs cardiac risk assessments to determine if there is a need for lipid-lowering therapy. If antiretroviral drug therapy proves ineffective for a particular patient, the pharmacist interprets the reports of HIV drug resistance and recommends new regimens based on drug resistance mutations.

At the time of writing, in spring 2009, almost 1400 patients were being followed through the Northern Alberta HIV Program, and this number continues to grow. Dr Hughes devotes about 2 days per week to the HIV program, working at the University of Alberta hospital site. In 2001, a second site 
was opened at an inner-city hospital, and a 0.5 full-time equivalent (FTE) pharmacist position was established through funding from Province Wide Services. Province Wide Services is in turn funded by Alberta Health and Wellness and covers antiretroviral drugs, as well as program delivery for HIV care in the province of Alberta. In 2006, the Northern Alberta HIV Program formally requested and received funding from Province Wide Services for 2 additional FTE pharmacists, who are shared between the 2 sites. The case for increased pharmacist funding was based on increases in patient load (demonstrated by workload statistics) and also recognition of the pharmacist's role in the management of medication therapy, which is an important component of treating HIV-related disease.

This ambulatory practice area provides a good example of the importance of interdisciplinary care in serving a patient population with complex needs. It serves as an excellent training opportunity for undergraduate and Doctor of Pharmacy students and for residents with respect to understanding the pharmacist's role within a health care team. It also provides an opportunity for students to further develop their history-taking, patient assessment, problem-solving, and communication skills in a team environment. The recent establishment of prescriptive authority in Alberta has allowed continued expansion of the pharmacist's clinical role in the Northern Alberta HIV Program, with the goal of improving the delivery of patient care in a team environment.

The Practice Spotlight series highlights the accomplishments of Canadian pharmacists with unique practices in hospitals and related health care settings. If you have a unique or innovative practice, or you know someone else who should be profiled, please submit your contact information to Mary Ensom, Editor of CJHP (cjhpedit@cshp.ca), and one of our Associate Editors will be in touch with you.

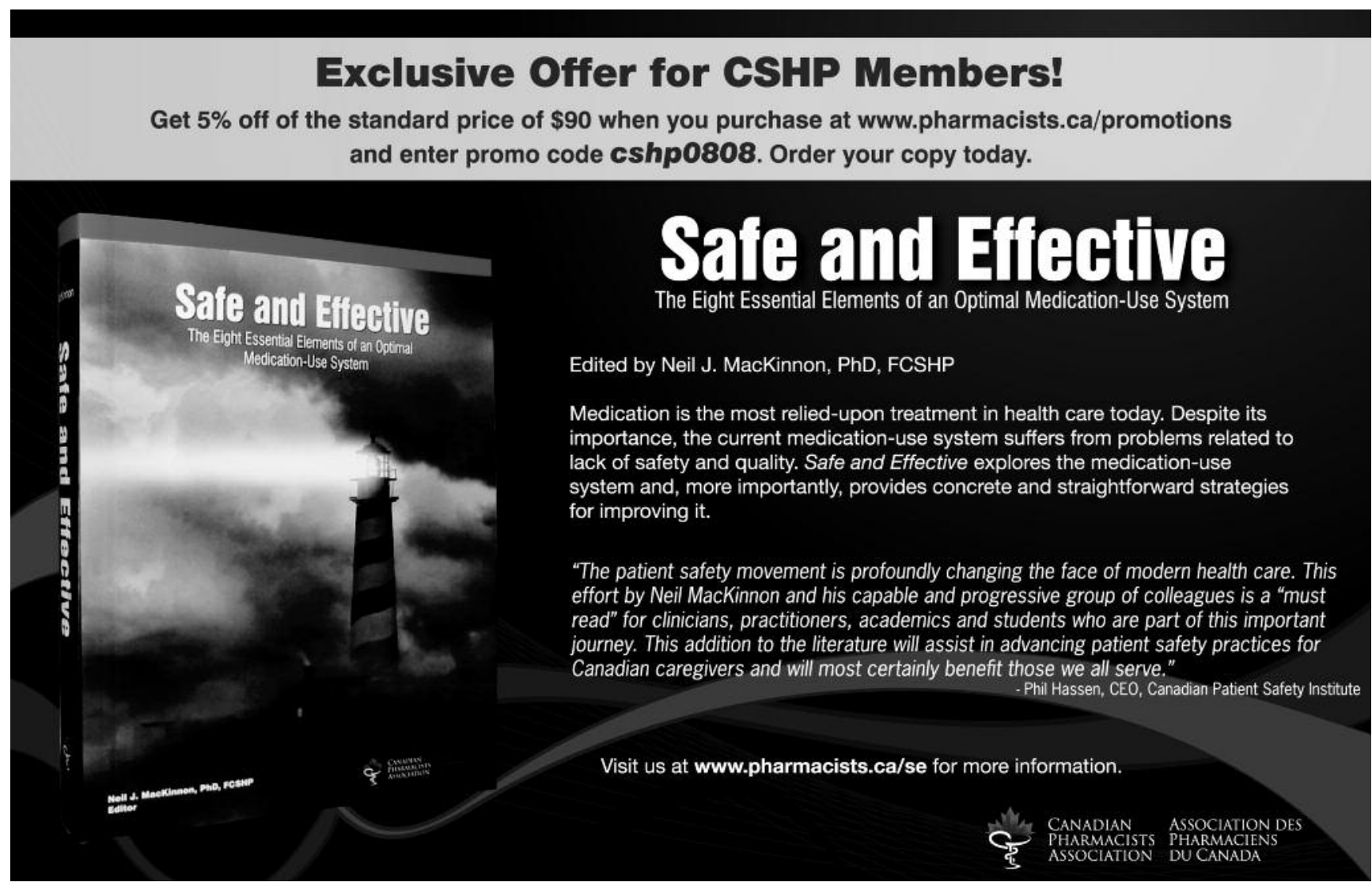

